\title{
O TRANSPORTE DE DIAMANTES POR SISTEMAS FLUVIAIS A LONGAS DISTÂNCIAS: UMA VISÃO CRÍTICA
}

\author{
JOSÉ ELOI GUIMARÃ ES CAMPOS* \& GUILHERME MODESTO GONZAGA**
}

\begin{abstract}
THE TRANSPORT OF DIAMOND BY FLUVIAL SYSTEMS FOR LONG DISTANCE: A CRITICAI VIEW Many authors believe in the possibility of diamond transport for hundreds of kilometers by fluvial sedimentary processes. This work attempts to show by the integration of alluvial placer observations, experimental data analysis and case study that diamonds cannot be transported for long distances by tractive processes. The presence of diamond in a large geographical area (hundreds of square kilometers) implies in the necessity of the presence of multiple well distributed sources, including primary (kimberlite and lamproite) or secondary (sedimentary rocks) sources. The coarse facies related to the glaciogenic sediments are the most important sources among the secondary host rocks. The case studies exemplified in this paper show clearly that the wide dispersion of diamonds is mainly explained by the presence of secondary sources with regional distribution.

Keywords: fluvial transport, diamond, placers, facies.

RESUMO - Vários autores acreditam na possibilidade de transporte de diamantes por centenas de quilómetros através de ambientes fluviais. Este trabalho tenta mostrar a partir da integração da observação de placeres aluvionares, de dados experimentais e de estudo de casos, que diamantes não podem ser transportados por processos tracionais a longa distâncias. A presença de diamantes em uma extensa região (milhares de quilómetros quadrados) implica na necessidade da presenca de numerosas fontes distribuídas por toda a bacia, podendo ser representadas por fontes primárias (kimberlitos ou lamproítos), secundárias (rochas sedimentares) ou ambas. Entre os hospedeiros secundários, as fácies rudíticas relacionadas aos sedimentos glacio gênicos são destacados pelos autores. $\mathrm{O}$ estudo dos casos exemplificados neste trabalho mostra claramente que a grande dispersão de diamantes é devida principalmente a presença de fontes secundárias com ampla distribuição regional.

Palavras-chave: transporte fluvial, diamante, placeres, fácies.
\end{abstract}

INTRODUÇÃO Diversos autores apresentam uma visão distorcida a respeito do transporte de minerais pesados por sistemas fluviais. Como exemplos, podemos citar Wagner \& Merensky (1928) e Hallam (1964), os quais consideram que os diamantes presentes na costa atlântica sul-africana foram transportados pelo Rio Orange desde a região de Kimberley, a uma distância superior a $1000 \mathrm{~km}$ no interior do continente, ou Ambroise (1991) que propõe um transporte de centenas de quilometros para os diamantes observados em cursos fluviais no nordeste de Angola.

A análise dos processos fluviais integrada a partir de: observações de campo, análise dos principais condicionantes para origem de placeres, resultados experimentais de laboratório e estudo de casos, mostra que os minerais pesados de forma geral e os diamantes em particular apresentam curto transporte em ambiente fluvial e uma tendência a rápida deposição.

Algumas características destes depósitos secundários valorizam seu potencial exploratório, tais como a natureza inconsolidada que viabiliza economicamente depósitos com teor de alguns pontos por metro cúbico; o relativo baixo custo de exploração, pesquisa e lavra; a possibilidade de se desenvolver uma lavra em circuito hidráulico fechado (para reciclagem de água), que minimizam os impactos ambientais e a alta razão entre as gemas e os diamantes industriais, aumentando os precos médios por quilate.

Outro aspecto importante é o fato de que a localização de jazidas primárias frequentemente passa peto estudo e entendimento das ocorrências secundárias em sistemas fluviais.

O objetivo deste trabalho é rediscutir a questão, geralmente aceita, a respeito da distribuição de diamantes a centenas de quilómetros de sua fonte, por sistemas fluviais. Também objetiva mostrar como se processa o transporte de elementos pesados por sistemas fluviais, para contribuir aos aspectos práticos a respeito da geração de placeres e/ou paleoplaceres e para a prospecção de depósitos primários e secundários de diamante

MECANISMOS DE TRANSPORTE FLUVIAL Para o entendimento dos mecanismos de transporte do diamante a partir de suas fontes primárias conhecidas (províncias kimberlíticas e lamproíticas), serão tecidas considerações gerais sobre os processos fluviais capazes de transportar minerais pesados. Os ambientes, onde são encontrados depósitos diamantíferos, incluem os sistemas fluviais de leques aluviais, de rios entrelacados, de rios meandrantes e os depósitos resultantes da interação destes sistemas. Ambientes fluvio-glaciais e canais subglaciais (zona basal de geleiras), também apresentam elevado potencial para acumulação de minerais pesados, sendo as fácies sedimentares de maior regime de fluxo conhecidas; e.g. fácies de rios entrelaçados tipo Scott, (Miall 1978) e canais de alta pressão em bases de geleiras (Eyles 1995).

Leques aluviais As fácies fluviais do tipo "leques aluviais" são caracterizadas por uma sedimentação proximal grossa com correntes aquosas e fluxos de detritos associados. Em função do clima vigente, podem ser classificados como leques semi-áridos (com predominância de processos de transporte por debrís flow) ou leques tímidos (com contribuição de transporte por tração). Sua implantação depende de altos topográficos, pois necessitam gradiente de relevo acentuado, podendo o mesmo ser originado ao longo de escarpas de falhas, por soerguimento crustal ou a partir da construção de edificios vulcânicos. As litologias depositadas incluem desde conglomerados polimíticos (com proporções variáveis de matriz) até arenitos e argilitos nas porções distais. Entre as estruturas sedimentares observadas ocorrem canais erosivos, acamamento gradacional e estratos cruzados acanalados. A espessura dos depósitos é diretamente controlada pela magnitude do soerguimento nas áreas adjacentes. No caso de regiōes com atividade tectônica prolongada, depósitos de até milhares de metros de espessura podem ser registrados (e.g. Bacia do Recôncavo-TucanoJatobá).

Um exemplo de depósito de diamante originado em condições de leques aluviais é a antiga "mina" de Romaria no oeste do estado de Minas Gerais (Suguio et al 1979). Os minerais pesados, neste caso, são concentrados nas bases de canais de corte e preenchimento e mais comumente intertrapeados entre os seixos e blocos.

Sistemas de rios entrelaçados $\mathrm{O}$ sistema fluvial entrelaçado é caracterizado pela alta energia de transporte em regime de fluxo superior. E caracterizado pela migracão de barras longitudinais e transversais com deposição de cascalhos, areias e muito raramente material pelítico (apresentando pequena distribuição lateral). Dependendo da natureza do suprimento, do gradiente a partir das áreas fontes e da razão de descarga (velocidade de fluxo e carga de sedimentos), ocorrem rios entrelaçados dominados por areias ou por cascalhos. Os rios dominados por cascalhos são mais importantes na concentração de minerais pesados, sendo representados por sub-canais divididos por barras emersas nas épocas secas. A migração destas formas de leito produz conglomerados e arenitos seixosos com imbricação de seixos, estratos cruzados, acamamento gradacional normal e inverso, além de canais de reativação. $\mathrm{O}$ transporte se dá predominantemente através de processos tracionais e existe uma tendência de diminuição da granulometria com o transporte. Leopold et al. (1992) consideram que o aumento de granulometria e a presença de novos materiais na carga de fundo são causados pela entrada de distributários, com diferente história deposicional, do sistema entrelaçado principal.

Um exemplo de depósito auri-diamantífero, originado a partir de um sistema fluvial entrelaçado, é representado pelas ocorrências de ouro e diamante na região do Rio Mau no estado de Roraima (Pessoa \&D'Antonnal988).

Rios meandrantes Os sistemas de rios meandrantes são caracterizados por grande carga em suspensão e reduzido transporte de materiais finos por tração (areias e silte), portanto não representam um sistema importante para a geração de placeres. 
Contudo, como observado por Miall (1977), as características dos vários tipos de fácies fluviais podem se superpor no tempo e no espaço. Dessa forma, os depósitos tipo placeres observados em cursos de rios meandrantes atuais, foram na realidade gerados em um intervalo de tempo anterior, quando o rio apresentava um canal mais retilíneo do tipo entrelaçado, com importante transporte e deposição de cascalho (atualmente observados na base dos canais). Este período representa uma.fase associada a um estágio geomorfológico juvenil da evolução fluvial.

Um exemplo de um sistema meandrante atual com ocorrências diamantíferas tipo placer é representado por segmentos de grande extensão do Rio Paranaíba no Triângulo Mineiro.

GERAÇÃO DE PLACERES FLUVIAIS Os principais controles para a geração de depósitos do tipo placeres incluem a presença de minerais de valor económico na área fonte, transporte em regime de fluxo superior e sua acumulação nos ambientes mais favoráveis. Em síntese, a deposição e a concentração de minerais pesados estão diretamente relacionadas às variáveis hidráulicas, sendo então previsíveis as condições e locais ideais para sua deposição, bem como os ambientes, sub-ambientes e fácies mais propícias.

Entre os parâmetros associados ao fluxo mais importantes que controlam a geração dos placeres se destacam: a intensidade e variabilidade da descarga; a quantidade e tamanho dos clastos transportados por tração; a velocidade da corrente; o gradiente do sistema; a quantidade e tipo de vegetação; o clima; o tipo de fonte (primária ou secundária), o arcabouço tectônico e a evolução geomorfológica da área.

Outros fatores locais também controlam a acumulação de minerais pesados pelos sistemas fluviais:

A - aprisionamento de minerais pesados entre os seixos: grãos de minerais que deveriam ser transportados por determinadas correntes (e.g. que transportam clastos de quartzitos de $40 \mathrm{~cm}$ ) são capturados pela armadilha gerada pelos espaços entre os seixos maiores (Muggeridge 1986);

B -junção de sistemas de drenagem com regimes de fluxo diferentes: o material transportado por sal tacão se deposita por agradação vertical quando alcança outro sistema de fluxo de menor competência (Mosley \& Schumm 1977);

C - descontinuidades no bedrock: as barreiras caracterizadas por heterogeneidades na forma do leito (fraturas, xistosidades, contatos, diques, etc.), constituem pontos preferenciais para acúmulo de minerais pesados a partir da remoção dos leves devido ao turbilhonamento da corrente;

D - sinuosidade dos canais: as porções internas de curvas de canais principais de rios meandrantes, ou de barras de sistemas entrelaçados, são locais preferenciais para a deposição, pois constituem sítios de rápida queda de energia (Smith \& Beukes 1983);

E - base de canais de reativação: funcionam como "embasamentos intraformacionais". Neste caso, em uma sucessão de cascalhes, com constantes canais de corte e preenchimento, podem ocorrer várias camadas ricas em minerais pesados, ao contrário daquelas referentes a conglomerados basais onde a concentração está apenas associada ao bedrock;

$\mathrm{F}$ - regiões de grande gradiente local: corredeiras e depressões também promovem um turbilhonamento do fluxo, gerando pontualmente uma grande concentração de minerais pesados.

G - gradiente do embasamento: condições de pequena inclinação do embasamento favorecem o rápido aprisionamento dos minerais pesados (Day \& Fletcher 1991);

H - canais abandonados: o isolamento de canais devido a migração lateral das formas de leito constituem locais preferenciais para acumulação em função da rápida diminuição de energia.

A figura 1 ilustra de forma esquemática os controles mostrados anteriormente.

Os processos descritos permitem considerar algumas feições petrográficas importantes na caracterização de fácies de cascalhes e de conglomerados, que são mais favoráveis à geração de placeres diamantíferos. Dentre os controles petrográfícos, devem ser levados em consideração:

- a análise qualitativa e quantitativa dos minerais pesados, já que o teor em diamantes é diretamente proporcional a concentração dos minerais pesados;

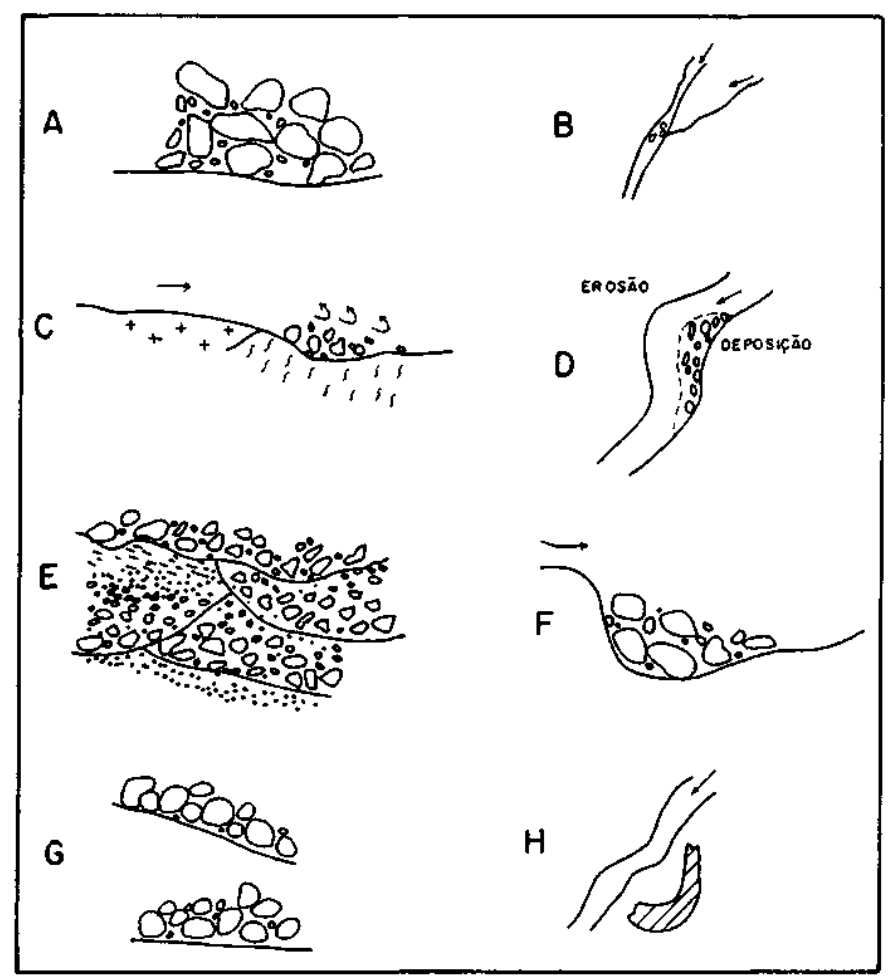

Figura l - Processos favoráveis à geração de acumulações diamantiferas do tipo placeres fluviais. I e II se referem ao gradiente do embasamento. Discussões mais detalhadas no texto.

- a análise de frequência granulométrica, principalmente a caracterização dos maiores clastos, uma vez que existe uma relação direta entre granulometria dos materiais fluviais e teor em diamantes;

- a caracterização da quantidade de matriz, pois existe uma relação inversa entre teor de diamantes e quantidade de matriz;

Como os controles locais mostrados anteriormente são observados com grande frequência nos sistemas fluviais naturais (variações granulométricas, irregularidades no embasamento, rápida mudança da velocidade de fluxo, etc.), fica claro que a tendência dos minerais pesados é sofrer um pequeno transporte fluvial, portanto sendo pouco provável o transporte de diamantes a centenas de quilómetros de distância da área fonte por este tipo de agente transportador.

DADOS EXPERIMENTAIS Os mecanismos de transporte e deposição de minerais pesados têm sido motivo de estudos experimentais por vários autores (Minter \& Toens 1970, Slingerland 1977, Kuhnle \& Southard 1990, entre outros). Os trabalhos experimentais convergem para os mesmos resultados, indicando que os minerais pesados tendem a se concentrar em uma camada basal (heavy infralayer) enquanto os leves acumulam-se posteriormente (light supralayer). Tal disposição é sempre alcançada quando se transporta misturas de minerais pesados e leves por correntes aquosas em calhas (flume experiments).

Nos sistemas naturais, os minerais pesados também se acumulam na base das sequências sedimentares, preferencialmente próximo ao bedrock ou nas porções basais de fluxos individuais. A deposição dos minerais pesados é previsível em função de processos hidráulicos que relacionam as características fisicas das correntes (velocidade e viscosidade do fluxo) com as características físicas dos materiais transportados (diâmetro e densidade).

Rombouts (1995) através de estudos de estatística experimental, conclui não ser possível um transporte de grandes diamantes em regimes fluviais por longas distâncias. Este autor, analisando dados de vários depósitos, atribui a presença de inúmeras fontes (mistura de fontes primárias distintas ou confluência de sistemas fluviais) para o fato da não aplicabilidade da distribuição lognormal (observada para a maioria dos sistemas geológicos) para alguns depósitos diamantíferos.

Hattingh \& Rust (1993) realizaram um trabalho experimental, onde 40 toneladas de areia de praia rica em ilmenita, granada, perovskita e limonita foram acumuladas a montante de uma sinuosidade do curso do Rio Swartkops (África do Sul). Após um período de fluxo intenso, 
os minerais pesados acumularam-se na porção interna da curva do rio e não sofreram transporte superior a algumas centenas de metros.

Sutherland (1982) propôs juma "curva de seleção" para o diamante transportado por processos fluviais, objetivando descrever a variação de tamanho dos diamantes com a distância da área fonte. A curva que descreve a equação, tem a forma geral de uma exponencial:

$\mathrm{y}=\mathrm{a} \cdot \mathrm{e}^{\{-} \mathrm{bx} \mathrm{x}^{\left.\left(1^{\circ / 2}\right)\right]}$

onde y é o tamanho médio da pedra a uma distância $\mathrm{x}$ da área fonte, $\mathrm{x}$ a distância medida em quilómetros e a e b são parâmetros determinados pelo método dos mínimos quadrados (respectivamente tamanho médio das pedras na área fonte e constante de desintegração que varia de $0,10 \mathrm{a}$ 0,20 nos sistemas naturais).

A análise numérica da equação mostra que com um transporte fluvial de cerca de $500 \mathrm{~km}$ uma pedra originalmente de 10 quilates teria cerca de 0,035 quilates (3,5 pontos - tamanho aproximado de um grão de areia grossa) e que uma pedra de cerca de 3,7 quilates (em um aluvião) deveria apresentar 100 quilates na área fonte após um transporte de $400 \mathrm{~km}$. Este fato mostra que mesmo uma equação teórica obtida através de análises empíricas, não sustenta a ideia de um longo transporte pelos sistemas fluviais, pois diamantes de dezenas de quilates são relativamente comuns em vários depósitos secundários. Os grandes diamantes localizados a centenas de quilómetros das fontes primárias certamente não foram distribuídos apenas por sistemas fluviais, sendo bastante provável, em alguns casos, a atuação de processos glaciogênicos (Gonzaga \& Tompkins 1991 e Campos et al. 1993).

As "curvas de calibração", que representam as tendências de diminuição dos diamantes com o avanço do transporte fluvial, são utilizadas como metodologia adicional para a localização das áreas fontes. A tentativa de aplicação de curvas de calibração para populações de diamantes muito distantes da fonte, frequentemente mostra variações erráticas na distribuição dos tamanhos dos diamantes, indicando certamente, outras fontes ao longo do curso fluvial (Rouffaer 1988).

Outra situação real, que também favorece a tese de uma rápida acumulação dos minerais pesados pode ser observada nos sistemas de garimpagem artesanal desenvolvidos no Brasil. Neste caso, os cascal-hos são concentrados em calhas de metal ou de madeira, com barreiras transversais, que apresentam comprimento médio de 5 metros. A experiência dos garimpeiros mostra não haver qualquer prejuízo na lavra (quando desenvolvida de maneira apropriada), mesmo considerando as reduzidas extensões das calhas, ficando todos os diamantes retidos nos primeiros metros e não ocorrendo registros de perdas, sequer das pedras mais finas. $\mathrm{O}$ mesmo não é válido para a concentração de ouro em sistemas de calhas similares. Os fragmentos de ouro com formas em palhetas (devido a sua maleabilidade), ao contrário dos diamantes, apresentam forte interferência de processos suspensivos durante seu transporte em regimes fluviais.

Mesmo considerando a atuação de sucessivos ciclos de transporte fluvial, e não o transporte em um instante de tempo, como até então estabelecido, não se deve esperar a transferência de diamantes por centenas de quilómetros em uma bacia sedimentar, uma vez que o contraste de densidade entre o diamante $(\rho=3,5)$ e os materiais componentes dos cascalhes fluviais (média de $\rho=2,75$ ) é significativo, resultando em uma tendência geral para a deposição destes minerais. O único ambiente deposicional capaz de transportar diamantes por distâncias consideráveis é o sistema glacial, que através do transporte de grande volume de massa pela expansão das capas de gelo, pode ser responsável pela transferência de material por até centenas de quilômetros. Flint (1971) mostra um transporte de matacões numa distância de $1200 \mathrm{~km}$, desde o Escudo Canadense até a região central dos Estados Unidos. Já Duskin \& Jarvis (1993) consideram o transporte de diamantes por centenas de quilómetros, desde o estado de Michigan, até Wisconsin nos EUA.

ANÁLISE DE CASOS Objetivando exemplificar as idéias discutidas nesta contribuição, foram escolhidos dois exemplos de depósitos diamantíferos associados a sistemas fluviais bastante difundidos na literatura. Os exemplos escolhidos são representados pelos depósitos diamantíferos da região da Serra do Espinhaço Meridional, incluindo os aluviões do Rio Jequitinhonha, no estadç de Minas Gerais, e pelos depósitos diamantíferos do Rio Orange, na África do Sul e na Namíbia, bem como pelos placeres diamantíferos da costa atlântica destes países.

Rio Jequitinhonha (Brasil) O Rio Jequitinhonha está localizado no nordeste do Estado de Minas Gerais, em uma região que durante o século XVIII foi responsável pela maior produção mundial de diamantes. As ocorrências diamantíferas estão distribuídas por cerca de 400 quilómetros ao longo do curso fluvial. Neste trecho do rio existe uma marcante variação do tamanho médio das pedras recuperadas dos aluviões e paleoaluviões cenozóicos.

O contexto geológico, para efeito deste trabalho, é representado pelas seguintes unidades: Supergrupo Espinhaço, Grupo Macaúbas (subdividido nas fácies glacio-continentais e transicionais proximais e nas fácies glacio-marinhas proximais e marinhas distais), Sequências vulcano-sedimentares e Grupo Bambuí. A distribuicão espacial das unidades e sua relação com o curso fluvial são mostradas na figura 2. O Rio Jequitinhonha apresenta em sua maior extensão um regime fluvial do tipo meandrante, contudo as fácies grossas situadas na base do canal ativo e no fundo de todo $o$ flat (de até $2 \mathrm{~km}$ de extensão lateral) caracterizam um paleosistema entrelaçado, relacionado ao início de implantação da rede de drenagem.

As principais fontes diamantíferas, consideradas para a região, são os dois conjuntos litoestratigráficos, denominados de Formação Sopa Brumadinho e Grupo Macaúbas. A presença de diamantes nos metaconglomerados da Formação Sopa Brumadinho é consensual. Por outro lado, apenas alguns autores consideram o Grupo Macaúbas como uma importante fonte de diamantes (Derby 1906, Guimarães 1965, Gonzaga \& Dardenne 1991 e outros).

A Formação Sopa Brumadinho é posicionada na base do Grupo Espinhaço e é composta por quartzitos e metaconglomerados atribuídos a paleoleques aluviais e fácies transicionais, incluindo depósitos do tipo storm beach. O Grupo Macaúbas representa os sedimentos glaciogênicos de fácies glacio-continentais e transicionais correspondentes às porções proximais da glaciação, bem como as fácies plataformais e marinhas distais. As fontes primárias não foram identificadas na região, contudo considera-se que são representadas por eventos magmáticos Paleoproterozóicos no Cráton do São Francisco, os quais foram, em parte, possivelmente recobertos por sedimentos proterozóicos mais jovens (e.g. Grupo Bambuí).

O Rio Jequitinhonha é diamantífero desde sua nascente, onde drena metaconglomerados da Formação Sopa-Brumadinho, sendo as ocorrências de diamantes distribuídas onde o curso fluvial intercepta as rochas do Supergrupo Espinhaço e do Grupo Macaúbas (e.g. região da Mineração Rio Novo). No momento em que o rio flui sobre as fácies distais do Grupo Macaúbas, a presença de diamantes diminui sensivelmente, desaparecendo completamente nos trechos sobre as rochas granito-gnáissicas do embasamento cristalino. As ocorrências diamantíferas são apenas observadas nas regiões proximais aos hospedeiros secundários, sendo sua ampla distribuição relacionada à grande dispersão das fontes secundárias.

A simples observação dos dados de variação de peso médio dos diamantes ao longo de mais de 250 quilómetros do Rio Jequitinhonha (Haralyi et al. 1991) permite concluir que o sistema fluvial foi responsável por um transporte reduzido. Segundo o gráfico da figura 3, que mostra a variação de peso (tamanho) de diamantes por distância de transporte em quilómetros, existe um rápido incremento do tamanho das pedras em três regiões do vale fluvial: próximo a Mendanha, na foz do Rio Coité Mirim e na foz do Rio Macaúbas (respectivamente 1, 2 e 3 destacados com setas na figura 2). Estas três localidades representam marcantes pontos de entradas de novos diamantes, a partir de fontes diversas, e não um transporte contínuo a partir do Alto Rio Jequitinhonha (nas cabeceiras do rio). A integração dos dados geológicos com os dados de variação de peso das pedras, pode explicar satisfatoriamente os bruscos aumentos no peso mediano dos diamantes: - na região 1 existem amplas áreas de afloramento de metaconglomerados do Grupo Espinhaço (principal hospedeiro secundário); na região 2 as fontes locais são atribuídas aos metaconglomerados da Formação Sopa Brumadinho e aos diamictitos do Grupo Macaúbas e na região 3, onde o Rio Macaúbas flui sobre grande área de diamictitos glacio-continentais, que representam fácies potencialmente portadoras de diamantes (Rouffaer 1988).

A partir da análise anterior, foi possível mostrar que o Rio Jequitinhonha é mais um exemplo de um sistema natural que demonstra claramente o transporte de diamantes a pequenas distâncias por sistemas fluviais. O mesmo raciocínio pode ser desenvolvido para as ocorrências secundárias oriundas dos kimberlitos da região de Juína/MT, onde os placeres, via de regra, se distribuem no máximo a dezenas de quilómetros das fontes primárias.

Rio Orange (África do Sul) Inúmeros autores acreditam que a origem dos diamantes do Rio Orange, na África do Sul, e dos depósitos diamantíferos na costa atlântica da África do Sul e Namíbia, esteja ligada a fontes primárias localizadas no Cráton de Kapvaal, distantes a cerca de 1200 quilómetros. Rouffaer (1988) e Marshall \& BaxterBrown (1995), ao contrário, afirmam que a grande maioria destes 


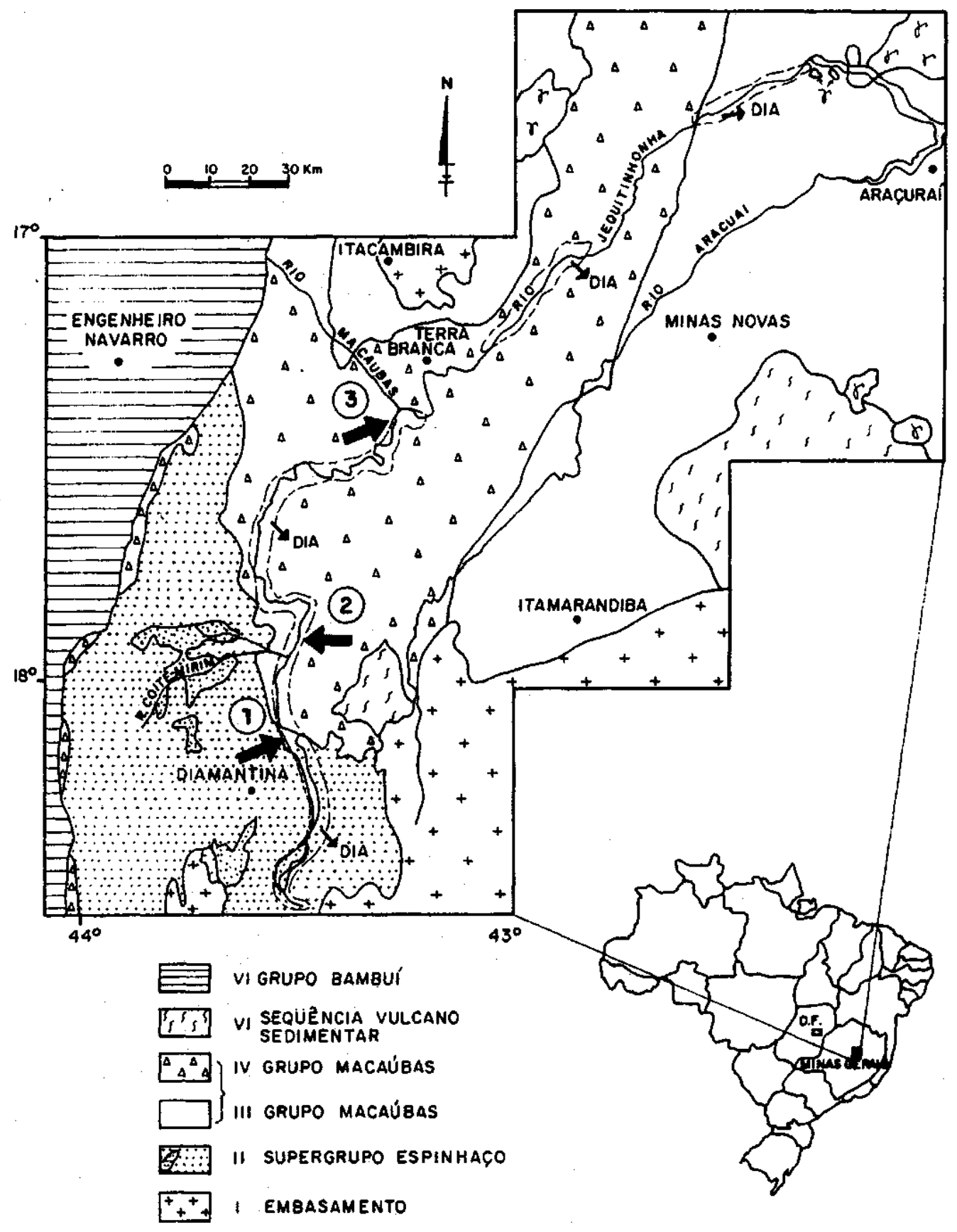

Figura 2-I- Embasamento: gnaisses de composição granítica, II - Supergrupo Espinhaço: quartzitos e conglomerados continentais e sequências psamo-pelíticas litorâneas e marinhas. III - Grupo Macaúbas: fácies glacio-marinhas e turbidíticas. IV - Grupo Macaúbas: fácies glacio continentais e transicionais. $\boldsymbol{V}$-Sequências Vulcano-Sedimentares: rochas metavulcânicas ultrabásicas, básicas e intermediárias e meta sedimentos químicos e pelíticos. VI - Grupo Bambut: metassedimentos pelíticos e carbonáticos. _ - granitos intrusivos. (adaptado de Pedrosa-Soares et al. 1994).

diamantes são derivados de rochas sedimentares.glaciogênicas permocarboníferas relacionados à Formação Dwyka.

O teor geral de diamantes nos sedimentos Dwyka é bastante baixo. Porém, assumindo a presença de menos de um quilate por milhões de toneladas de sedimento, depósitos resultantes da erosão e reconcentração de bilhões de toneladas destes sedimentos poderiam explicares depósitos secundários do Rio Orange, como também da costa atlântica (Marshall \& Baxter-Brown 1995). Na realidade, os diamantes do Rio Orange são oriundos de diversos afloramentos de sedimentos Dwyka (fontes secundárias) e foram transportados por alguns quilómetros, sendo retidos por diversos tipos de armadilhas fluviais. E muito importante citar que a região da foz do Rio Orange apresenta uma mediana de peso dos diamantes superior àquela observada no seu alto curso (Rombouts 1995). Tal fato não é compatível com a distribuição de tamanhos de diamantes transportados em regimes fluviais e vem reforçar a presença de várias fontes secundárias ao longo do curso fluvial.

A figura 4, derivada a partir da superposição de ilustrações de Rouffaer (1988), mostra claramente uma estreita relação entre os sedimentos Dwyka, com as ocorrências diamantíferas do Rio Orange. Os depósitos aluvionares são constantes ao longo do médio curso do Rio Orange e desaparecem quando o rio seciona outros sedimentos e rochas do embasamento, reaparecendo próximo a foz do rio, onde novamente ocorrem grandes volumes de sedimentos glaciogênicos. E importante salientar que apesar da figura 4 não mostrar a existência da Formação Dwyka, ao longo da costa atlântica, esta está presente em amplas regiões formando falésias na margem continental (Rouffaer 1988). 


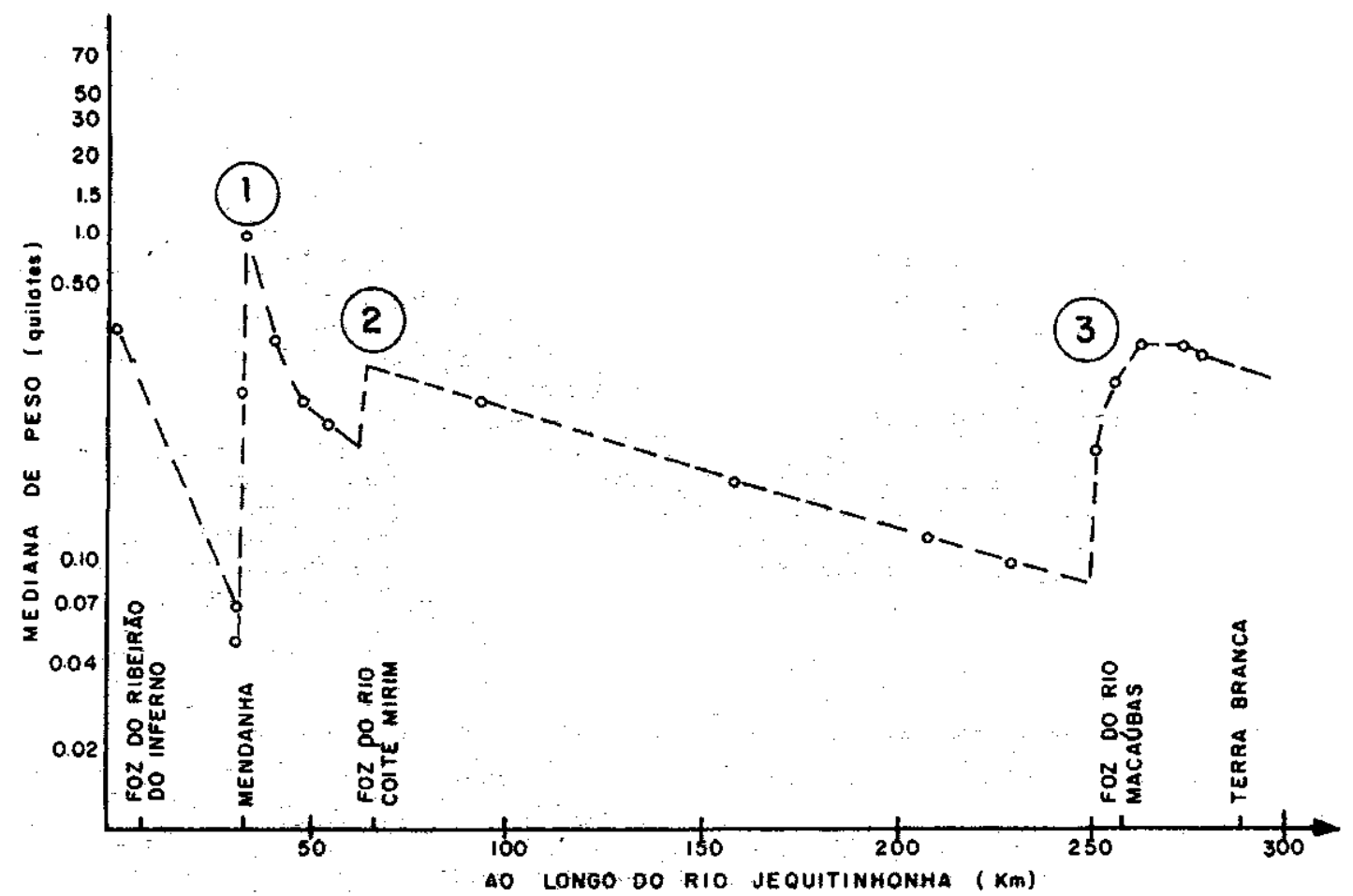

Figura 3 - Distribuição da mediana de peso de diamantes aluvionares ao longo do Rio Jequitinhonha (em escala logarítmica). A localização dos pontos 1, 2 e 3 estão mostrados na figura 2. (Compilado deHaraly et al. 1991).

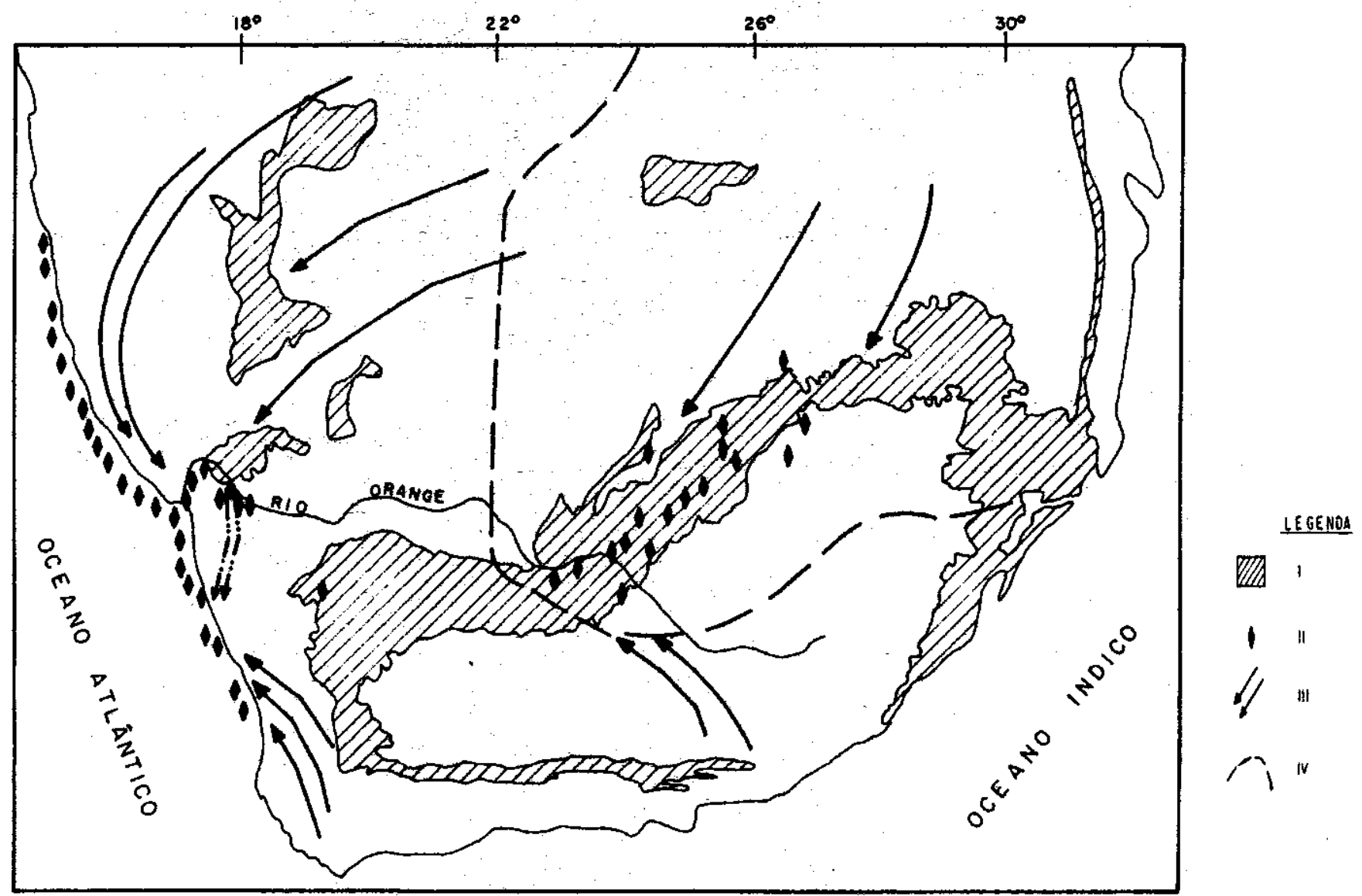

Figura 4 - Localização do Rio Orange na República dá África do Sul. I - Sedimentos glaciogênicos Permo-Carboníferos da Série Dwyka. II Depósitos aluvionares de diamante. III -Paleofluxo glacial. IV - Limite do Cráton de Kapvaal. 
Este exemplo, juntamente com o do Rio Jequitinhonha no Brasil, reforça significativamente dois pontos de vista que ainda se encontram pouco difundidos na literatura geológica:

- os processos glaciais são poderosos agentes de transporte e, em alguns casos, de concentração de diamantes.e outros minerais pesados;

- os sistemas fluviais não são agentes capazes de transportar diamantes a centenas de quilómetros de distância das fontes.

CONCLUSÕES O diamante, por ser um mineral pesado, cujas características físicas e químicas lhe conferem extrema resistência e estabilidade durante o transporte, pode ser retrabalhado a partir de suas fontes através de sucessivos ciclos deposicionais. domínio de transporte de cascalhes juntamente com fácies fluvioglaciais e canais subglaciais, são os agentes mais eficientes na concentração de minerais pesados.

- os sistemas fluviais transportam minerais pesados a distâncias reduzidas, geralmente não compatíveis com centenas de quilómetros da fonte, como usualmente é sugerido na literatura. A tendência natural maior energia.
- os sistemas deposicionais do tipo rios entrelaçados, com predo diamante é a rápida deposição nas fácies proximais nos ambientes de

- quando ocorrências diamantíferas são distribuídas por uma grande região, deve-se esperar a presença de várias fontes (primárias ou hospedeiros secundários) ao longo de todo o sistema fluvial, já que de uma forma geral, os minerais pesados não são transportados por longas distâncias em regimes fluviais. Estes minerais ficarão retidos nas várias modalidades de armadilhas nas regiões proximais das fontes. As fontes primárias diamantíferas apresentam uma dispersão de pequena magnitude, enquanto os hospedeiros secundários podem apresentar uma distribuição geográfica muito ampla.

- Dentre os possíveis hospedeiros secundários, que podem ser de diversos tipos, destaca-se os sedimentos glaciogênicos, que apresentam uma capacidade de transporte de centenas de quilómetros e em alguns casos apresentam condições de concentrar diamantes e outros minerais pesados.

- Este trabalho mostrou, a partir da integração de características do transporte fluvial, condicionantes de geração de placeres, dados experimentais e estudo de casos que os diamantes não são transportados por sistemas fluviais a longas distâncias, conforme usualmente descrito na literatura geológica. Este fato deve ser levados em consideração durante as campanhas exploratórias, pois a sua inobservância tem levado a equívocos de sérias consequências.

\section{Referências}

Ambroise, M. 1991. Geology of the NE Angolan Kimberlite region. In: Sth International Kimberlite Conference, CPRM, Extended Abstracts, 6-8.

Campos, J.E.G.; Dardenne, M.A. \& Gonzaga, G.M. 1993. O potencial diamantffero do conglomerado Abaete no NW de Minas Gerais. In: Simp. Brasil. Geol. Diamante. 1. Anais...Cuiabá. UFMT, Publ. Esp. 2/93. p. 101-113.

Day, S.J. \& Fletcher, W.K. 1991. Concentratiopn of magnetite and gold at bar and reach scales in a gravel-bed stream, British Columbia, Canada. Jour. Sed. Petrol., 61(6):871-882.

Derby, O. 1906. The Serra do Espinhaço, Brasil. Jour. Geol. , 14:374-401.

Duskin, D.J. \& Jarvis, W. 1993. Kimberlites in Michigan. In: Dunne, K.P.E. \& Grant, B. (ed.) Mid-Continent Diamonds. GAC-MAC Symposium Volume. Alberta. p. 105 $-106$

Eyles, N. 1995. Characteristics and origin of coarse gold in Late Pleistocene sediments of the Cariboo placer mining district, British Columbia, Canada. Sedimentar Geology, 95:69-95.

Flint, R.F. 1971. Glacial and Quaternary geology. John Willey \& Sons. New York. 892p.

Gonzaga, G.M. \& Dardenne, M.A. 1991. The Jequitái Glaciation and the dispersion of diamonds during Upper Proterozoic. In: 5th International Kimberlite Conference, Field Guide Book. CPRM. p. 89-93.

Gonzaga, G.M. \& Tompkins, L. A. 1991. Geologia do diamante. In: Principais Depósitos Minerais do Brasil. DNPM/CPRM. vol 4A. p. 53-116.

Guimarães, D. 1965. Princípios de metalogênese e geologia econômica do Brasil. MME/DNPM/DFPM. Rio de Janeiro. Boletim 121. 625pp.

Hallam, C. D. 1964. The geology of the coastal diamond deposits of Southern África. In: Haughton, S.H. (ed.), The geology ofsome ore deposits in Southern Africa II, Geol. Soe. S. África, p. 671-728.

Haralyi, N.L.E.; Hasui, Y. \& Morales, N. 1991. O diamante Pré-Cambriano da Serra do Espinhaço, Minas Gerais. In: Principais Depósitos Minerais do Brasil. DNPM/CPRM. vol 4A. p. 209-222.

Hattingh, J. \& Rust, I.C. 1993. Flood transport and deposition of tracer heavy minerais in a gravel-bed meander bend channel. Jour. Sed. Petrol., 63(5):828-834.

Kuhnle, R. A. \& Southard, J.B. 1990. Flume experiments on the transport of heavy minerais in gravel-bed streams. Jour. Sed. Petrol., 60(5):687-696.

Leopold, L.B.; Wolman, M.G. \& Miller, J.P. 1992. Fluvial processes in geomorjihology Dover Publications, Inc. New York. 522pp.

Marshall, T.R. \& Baxter-Brown, R. 1995. Basic principies of alluvial diamond exploration. Journal Geoch. Exploration, 53:277-292.
Miall, A.D. 1977. A review of the braided-river depositional environment. Earth Sci. Review, 13:1-62

Miall, A.D. 1978. Lithofacies types and vertical profiles in braided river deposits: a summary. Memoir of Canadian Society Petroleum Geologists, 5:597-605.

Minter, W.E.L. \& Toens, P.D. 1970. Experimental simulation of gold deposition in gravei beds. Geol. Soe. of South Africa Transactions, 73:89-99.

Mosley, M.P. \& Schummm, S.A. 1977. Stream junctions - a probable location for bedrock placers. Economic Geology, 72:691-697.

Muggeridge, M.T. 1986. The efficiency of fluvial trap sites to concentrate kimberlitic minerais: an experimental sampling programme. In: 4th International Kimberlite Conference, Geological Society of Austrália, Abstracts Series, 16:481-483. 1994. Nota esplicativa dos mapas geológico, metalogenético e de ocorrências minerais do Estado de Minas Gerais. Belo Horizonte. COMIG. 69p.

Pessoa, M.R. \& D'antonna, R.J.G. 1988. Depósitos auridiamantíferos do Rio Mau (RR) In: SBG, Congresso Brasileiro de Geologia, 35. Belém, 1988. Anais....Belém, SBG. Vol.1 p. 178-188.

Rombouts, L. 1995. Sampling and statistical evaluation of diamond deposits. Journal Geoch. Exploration, 53:351-367.

Rouffaer, E.J.H.F. 1988. On the origin of alluvial diamonds along the Atlantic Coast of southwest Africa. PhdThesis. Leuven. 155pp.

Slingerland, R.L. 1977. The effect of entrainment on the hydraulic equivalence relationships of light and heavy minerais in sands. Jour. Sed. Petrology, 47:753-770.

Smith, N.D. \& Beukes, N.J. 1983. Bar to bank flow convergence zones: a contribution to the origin of alluvial placers. Economic Geology, 78(7): 1342-1349.

Suguio, K; Svisero, D.P. \& Felitti, W. 1979. Conglomerados polimíticos diamantíferos de idade Cretácea de Romaria - MG: um exemplo de sedimentação de leques aluviais. In: Simp. Reg. Geol. 1. Arar....Rio Claro. p. 217-219.

Sutherland, D.G. 1982. The transport and sorting of diamonds by fluvial and marine processes. Economic Geology, 84(7):1613-1620.

Wagner, P.A. \& Merensky, H. 1928. The diamond deposits on the coast of Little Namaqualand. Trans. Geol. Soc. S. Africa, 31:1-41
Pedrosa-Soares, A.C.; Dardenne, M.A.; Hasui, Y.; Castro, F.D.C, e Carvalho, M.V.A. 